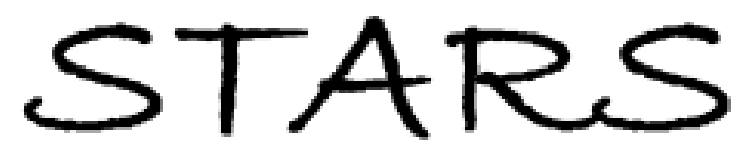

University of Central Florida

STARS

$1-1-2009$

\title{
Optimality Conditions for Semilinear Elliptic Equations With Leading Term Containing Controls
}

Hongwei Lou

Jiongmin Yong

University of Central Florida

Find similar works at: https://stars.library.ucf.edu/facultybib2000

University of Central Florida Libraries http://library.ucf.edu

This Article is brought to you for free and open access by the Faculty Bibliography at STARS. It has been accepted for inclusion in Faculty Bibliography 2000 s by an authorized administrator of STARS. For more information, please contactSTARS@ucf.edu.

\section{Recommended Citation}

Lou, Hongwei and Yong, Jiongmin, "Optimality Conditions for Semilinear Elliptic Equations With Leading Term Containing Controls" (2009). Faculty Bibliography 2000s. 1831.

https://stars.library.ucf.edu/facultybib2000/1831

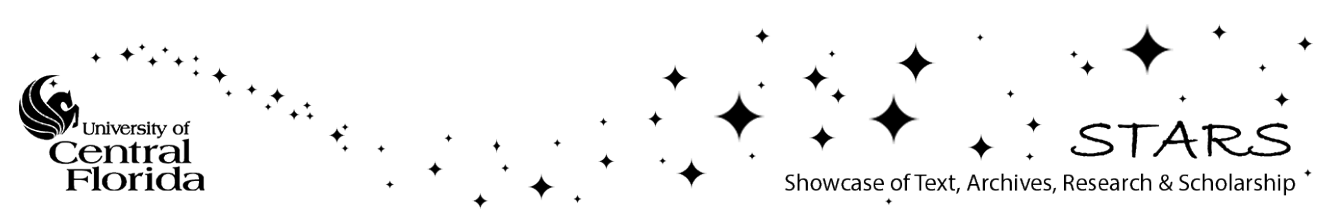




\title{
OPTIMALITY CONDITIONS FOR SEMILINEAR ELLIPTIC EQUATIONS WITH LEADING TERM CONTAINING CONTROLS*
}

\author{
HONGWEI LOU ${ }^{\dagger}$ AND JIONGMIN YONG
}

\begin{abstract}
An optimal control problem for semilinear elliptic partial differential equations is considered. The equation is in divergence form with the leading term containing the control. Necessary conditions for optimal controls are established by the method of homogenized spike variation. The key to such a method is to modify the usual spike variational technique by taking into account the homogenization techniques for elliptic equations, together with an idea from the theory of relaxed controls. Problems with state constraints are also discussed by further adding some well-known penalty arguments involving the application of Ekeland's variational principle and finite codimensionality of certain sets.
\end{abstract} tion

Key words. optimal control, necessary conditions, elliptic equation, homogenized spike varia-

AMS subject classifications. 49K20,35B27

DOI. $10.1137 / 080740301$

1. Introduction. Consider the following controlled elliptic partial differential equation $(\mathrm{PDE})$ of divergence form:

$$
\left\{\begin{array}{l}
-\nabla \cdot(A(x, u(x)) \nabla y(x))=f(x, y(x), u(x)) \quad \text { in } \Omega \\
\left.y\right|_{\partial \Omega}=0,
\end{array}\right.
$$

where $\Omega \subseteq \mathbb{R}^{n}$ is a bounded domain with a smooth boundary $\partial \Omega, A: \Omega \times U \rightarrow \mathbb{R}^{n \times n}$ is a map taking values in the set of all positive definite matrices, and $f: \Omega \times \mathbb{R} \times U \rightarrow \mathbb{R}$, with $U$ being a separable metric space. Function $u(\cdot)$, called a control, is taken from the set

$$
\mathcal{U} \equiv\{w: \Omega \rightarrow U \mid w(\cdot) \text { is measurable }\} .
$$

Under some mild conditions, for any $u(\cdot) \in \mathcal{U},(1.1)$ admits a unique weak solution $y(\cdot) \equiv y(\cdot ; u(\cdot))$, which is called the state (corresponding to the control $u(\cdot))$. The performance of the control is measured by the cost functional

$$
J(u(\cdot))=\int_{\Omega} f^{0}(x, y(x), u(x)) d x
$$

for some given map $f^{0}: \Omega \times \mathbb{R} \times U \rightarrow \mathbb{R}$. Our optimal control problem can be stated as follows.

Problem $(\mathrm{C})$. Find a $\bar{u}(\cdot) \in \mathcal{U}$ such that

$$
J(\bar{u}(\cdot))=\inf _{u(\cdot) \in \mathcal{U}} J(u(\cdot)) .
$$

*Received by the editors November 9, 2008; accepted for publication (in revised form) April 17, 2009; published electronically July 17, 2009.

http://www.siam.org/journals/sicon/48-4/74030.html

${ }^{\dagger}$ School of Mathematical Sciences, and LMNS, Fudan University, Shanghai 200433, China (hwlou@fudan.edu.cn). This author was supported in part by NSFC (10671040 and 10831007), FANEDD (200522), and NCET (06-0359).

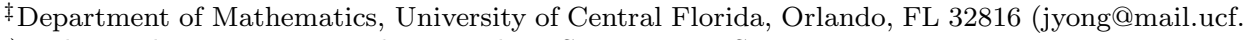
edu). This author was supported in part by NSF grant DMS-0604309.

2366

Copyright $@$ by SIAM. Unauthorized reproduction of this article is prohibited. 
Any $\bar{u}(\cdot) \in \mathcal{U}$ satisfying the above is called an optimal control, and the corresponding $\bar{y}(\cdot) \equiv y(\cdot ; \bar{u}(\cdot))$ is called an optimal state. The pair $(\bar{y}(\cdot), \bar{u}(\cdot))$ is called an optimal pair. In the case $A(x, u) \equiv A(x)$, Problem (C) has been studied by many authors; see [8] and the references cited therein. When $A(x, u)=u$ and $f(x, y, u)=f(x)$, the problem was studied in [5] for $U=[a, b]$ (with gradient constraint for the control), and was recently studied in [10] for

$$
U=M[\lambda, \Lambda] \equiv\left\{A \in \mathbb{R}^{n \times n} \mid A^{\top}=A, \lambda I \leq A \leq \Lambda I\right\}
$$

for some $\Lambda>\lambda>0$ with $f(\cdot)$ in $H^{-1}(\Omega)$; in [3] for $\mathcal{U}$ being some subset of

$$
\{A: \Omega \rightarrow M[\lambda, \Lambda] \mid A(\cdot) \text { is measurable }\},
$$

which is closed in the sense of $H$-convergence; and in [4] for $U=\{A, B\}$, where $A$ and $B$ are two given positive definite matrices and $f^{0}$ also depends on $\nabla y(\cdot)$. See, e.g., [6], [7], [9], [11], [12] for other relevant works.

In this paper, we make the following assumptions.

(S1) Set $\Omega$ is a bounded domain in $\mathbb{R}^{n}$ with a smooth boundary $\partial \Omega$.

(S2) Set $U$ is a separable metric space.

(S3) Function $A(x, v)=\left(a_{i j}(x, v)\right)$ takes values in the set $\mathcal{S}_{+}^{n}$ of $(n \times n)$ (symmetric) positive definite matrices, which is measurable in $x \in \Omega$ and continuous in $v \in U$. Further, there exist $\Lambda \geq \lambda>0$ such that

$$
\lambda|\xi|^{2} \leq\langle A(x, v) \xi, \xi\rangle \leq \Lambda|\xi|^{2} \quad \forall \xi \in \mathbb{R}^{n}, \quad \text { a.e. } x \in \Omega, v \in U,
$$

where $\langle\cdot, \cdot\rangle$ stands for the inner product in $\mathbb{R}^{n}$.

(S4) Function $f(x, y, v)$ is measurable in $x$ and continuous in $(y, v) \in \mathbb{R} \times U$ for almost all $x \in \Omega$. Moreover,

$$
f_{y}(x, y, v) \leq 0, \quad \text { a.e. }(x, y, v) \in \Omega \times \mathbb{R} \times U,
$$

and for any $R>0$, there exists an $M_{R}>0$ such that

$$
|f(x, y, v)|+\left|f_{y}(x, y, v)\right| \leq M_{R}, \quad \text { a.e. }(x, v) \in \Omega \times U,|y| \leq R .
$$

(S5) Function $f^{0}(x, y, v)$ is measurable in $x$ and continuous in $(y, v) \in \mathbb{R} \times U$ for almost all $x \in \Omega$. Moreover, for any $R>0$, there exists a $K_{R}>0$ such that

$$
\left|f^{0}(x, y, v)\right|+\left|f_{y}^{0}(x, y, u)\right| \leq K_{R}, \quad \text { a.e. }(x, v) \in \Omega \times U,|y| \leq R .
$$

Our main result is the following.

Theorem 1.1. Let (S1)-(S5) hold. Let $(\bar{y}(\cdot), \bar{u}(\cdot))$ be an optimal pair of Problem (C). Let $\bar{\psi}(\cdot)$ be the weak solution of the following adjoint equation:

$$
\left\{\begin{aligned}
-\nabla \cdot(A(x, \bar{u}(x)) \nabla \bar{\psi}(x))= & f_{y}(x, \bar{y}(x), \bar{u}(x)) \bar{\psi}(x) \\
& -f_{y}^{0}(x, \bar{y}(x), \bar{u}(x)) \quad \text { in } \Omega, \\
\left.\bar{\psi}\right|_{\partial \Omega}=0 . &
\end{aligned}\right.
$$

Then when $n=1$,

$$
\begin{array}{r}
H\left(x, \bar{y}(x), \bar{\psi}(x), \bar{y}^{\prime}(x), \bar{\psi}^{\prime}(x), \bar{u}(x)\right)-H\left(x, \bar{y}(x), \bar{\psi}(x), \bar{y}^{\prime}(x), \bar{\psi}^{\prime}(x), v\right) \\
\geq \frac{[A(x, v)-A(x, \bar{u}(x))]^{2}}{A(x, v)} \bar{y}^{\prime}(x) \bar{\psi}^{\prime}(x) \quad \forall v \in U, \quad \text { a.e. } x \in \Omega
\end{array}
$$

Copyright (c) by SIAM. Unauthorized reproduction of this article is prohibited. 
and when $n \geq 2$,

$$
\begin{gathered}
H(x, \bar{y}(x), \bar{\psi}(x), \nabla \bar{y}(x), \nabla \bar{\psi}(x), \bar{u}(x))-H(x, \bar{y}(x), \bar{\psi}(x), \nabla \bar{y}(x), \nabla \bar{\psi}(x), v) \\
\geq \frac{1}{2}\left|A(x, v)^{-\frac{1}{2}}(A(x, \bar{u}(x))-A(x, v)) \nabla \bar{y}(x)\right|\left|A(x, v)^{-\frac{1}{2}}(A(x, \bar{u}(x))-A(x, v)) \nabla \bar{\psi}(x)\right| \\
+\frac{1}{2}\left\langle A(x, v)^{-\frac{1}{2}}(A(x, \bar{u}(x))-A(x, v)) \nabla \bar{y}(x), A(x, v)^{-\frac{1}{2}}(A(x, \bar{u}(x))-A(x, v)) \nabla \bar{\psi}(x)\right\rangle \\
\forall v \in U, \quad \text { a.e. } x \in \Omega,
\end{gathered}
$$

where

$$
\begin{aligned}
H(x, y, \psi, \xi, \eta, v)= & \langle\psi, f(x, y, v)\rangle-f^{0}(x, y, v)-\langle A(x, v) \xi, \eta\rangle, \\
& (x, y, \psi, \xi, \eta, v) \in \mathbb{R}^{n} \times \mathbb{R} \times \mathbb{R} \times \mathbb{R}^{n} \times \mathbb{R}^{n} \times U .
\end{aligned}
$$

Note that the right-hand side of (1.10) is always nonnegative. Hence, (1.10) implies

$$
\begin{aligned}
& H(x, \bar{y}(x), \bar{\psi}(x), \nabla \bar{y}(x), \nabla \bar{\psi}(x), \bar{u}(x)) \\
& \quad=\max _{v \in U} H(x, \bar{y}(x), \bar{\psi}(x), \nabla \bar{y}(x), \nabla \bar{\psi}(x), v) \quad \forall v \in U, \quad \text { a.e. } x \in \Omega .
\end{aligned}
$$

However, the right-hand side of (1.9) could be negative. Also, it is not hard to see that when $A(x, v) \equiv A(x)$, the result automatically recovers those for the classical semilinear case without state constraints [8].

It is well known that since $U$ is not necessarily convex, one has to use spike variations to derive necessary conditions for optimal controls. Such a spike variation technique does not directly work for problems with leading term containing the control. To overcome the difficulty, we adopt the idea of homogenization for elliptic PDEs to carefully select some special spike variations of controls so that we can have desired "differentiability" of the state with respect to the control. We call such a newly developed approach the method of homogenized spike variation. It seems to us that this method can be used in treating many other relevant problems. We will report some further results along this line in our future publications.

The rest of the paper is organized as follows: In section 2, we present some preliminary results. Section 3 is devoted to a proof of our main result. A problem with state constraints will be discussed in section 4 . Some illustrative examples will be presented in section 5 .

2. Preliminaries. In this section, we will give some preliminary results needed in deriving necessary conditions for optimal controls. Recall that $\mathcal{S}_{+}^{n}$ is the set of all $(n \times n)$ (symmetric) positive definite matrices.

Proposition 2.1. Let (S1) hold. Let $B(\cdot)=\left(b_{i j}(\cdot)\right), C(\cdot)=\left(c_{i j}(\cdot)\right) \in L^{\infty}\left(\Omega ; \mathcal{S}_{+}^{n}\right)$ such that for some $\Lambda \geq \lambda>0$,

$$
\lambda|\xi|^{2} \leq\langle B(x) \xi, \xi\rangle,\langle C(x) \xi, \xi\rangle \leq \Lambda|\xi|^{2} \quad \forall \xi, x \in \mathbb{R}^{n} .
$$

Let $\delta \in(0,1)$ and let $g: \mathbb{R} \rightarrow \mathbb{R}$ be a periodic function with period 1 , and

$$
g(t)= \begin{cases}0, & t \in[0, \delta] \\ 1, & t \in(\delta, 1) .\end{cases}
$$

Copyright (C) by SIAM. Unauthorized reproduction of this article is prohibited. 
For $\varepsilon>0$, define

$$
B^{\varepsilon}(x)=B(x)+g\left(\frac{x_{1}}{\varepsilon}\right)[C(x)-B(x)] .
$$

Let $h(\cdot) \in L^{2}(\Omega)$, and let $y^{\varepsilon}(\cdot) \in H_{0}^{1}(\Omega)$ be the solution of

$$
\left\{\begin{array}{l}
-\nabla \cdot\left(B^{\varepsilon}(x) \nabla y^{\varepsilon}(x)\right)=h(x) \quad \text { in } \Omega \\
\left.y^{\varepsilon}\right|_{\partial \Omega}=0
\end{array}\right.
$$

Then

$$
y^{\varepsilon}(\cdot) \rightarrow y(\cdot), \quad \text { weakly in } H_{0}^{1}(\Omega),
$$

where $y(\cdot)$ is the weak solution of

$$
\left\{\begin{array}{l}
-\nabla \cdot(Q(x) \nabla y(x))=h(x) \quad \text { in } \Omega, \\
\left.y\right|_{\partial \Omega}=0,
\end{array}\right.
$$

with $Q(\cdot)=\left(q_{i j}(\cdot)\right) \in L^{\infty}\left(\Omega ; \mathcal{S}_{+}^{n}\right)$ given by

$$
\begin{gathered}
q_{i j}(x)=\delta b_{i j}(x)+(1-\delta) c_{i j}(x)-\frac{\delta(1-\delta)\left[b_{1 i}(x)-c_{1 i}(x)\right]\left[b_{1 j}(x)-c_{1 j}(x)\right]}{(1-\delta) b_{11}(x)+\delta c_{11}(x)} \\
1 \leq i, j \leq n
\end{gathered}
$$

The above proposition is a corollary of Theorem 2.2 in [1]. For the reader's convenience, we present a sketch of the proof here.

Sketch of the proof. Let $Z=[0,1]^{n}$. Define

$$
W(Z)=\left\{\varphi: \mathbb{R}^{n} \rightarrow \mathbb{R} \mid \varphi\left(z+e_{i}\right)=\varphi(z), \quad 1 \leq i \leq n, \forall z \in \mathbb{R}^{n}\right\},
$$

and let

$$
\begin{aligned}
A(x, z)=B(x)+g\left(z_{1}\right)[C(x)-B(x)] \equiv & \left(\alpha_{i j}\left(x, z_{1}\right)\right), \\
& x \in \Omega, z=\left(z_{1}, \ldots, z_{n}\right) \in \mathbb{R}^{n},
\end{aligned}
$$

with

$$
\alpha_{i j}\left(x, z_{1}\right)=b_{i j}(x)+g\left(z_{1}\right)\left[c_{i j}(x)-b_{i j}(x)\right], \quad 1 \leq i, j \leq n .
$$

Then (2.4) becomes

$$
\left\{\begin{array}{l}
-\nabla \cdot\left(A\left(x, \frac{x}{\varepsilon}\right) \nabla y^{\varepsilon}(x)\right)=h(x) \quad \text { in } \Omega, \\
\left.y^{\varepsilon}\right|_{\partial \Omega}=0 .
\end{array}\right.
$$

By [1, Theorem 2.2] (see also [2, Chapter 1, Theorem 6.1]), we have (2.5)-(2.6) with

$$
\begin{aligned}
q_{i j}(x)= & \int_{Z}\left\langle A(x, z) \nabla_{z}\left(\varphi^{i}(x, z)-z_{i}\right), \nabla_{z}\left(\varphi^{j}(x, z)-z_{j}\right)\right\rangle d z \\
= & \int_{Z}\left\langle A(x, z)\left(\nabla_{z} \varphi^{i}(x, z)-e_{i}\right), \nabla_{z} \varphi^{j}(x, z)-e_{j}\right\rangle d z \\
= & \int_{Z}\left[\alpha_{i j}\left(x, z_{1}\right)+\left\langle A(x, z) \nabla_{z} \varphi^{i}(x, z), \nabla_{z} \varphi^{j}(x, z)\right\rangle\right. \\
& \left.\quad-\left\langle A(x, z) \nabla_{z} \varphi^{i}(x, z), e_{j}\right\rangle-\left\langle A(x, z) \nabla_{z} \varphi^{j}(x, z), e_{i}\right\rangle\right] d z,
\end{aligned}
$$

Copyright (c) by SIAM. Unauthorized reproduction of this article is prohibited. 
where $\varphi^{k}(\cdot, \cdot)(k=1,2, \ldots, n)$ is the unique weak solution (up to an additive function of $x$ ) of

$$
\left\{\begin{array}{l}
-\nabla_{z} \cdot\left[A(x, z) \nabla_{z}\left(\varphi^{k}(x, z)-z_{k}\right)\right]=0 \\
x \mapsto \varphi^{k}(x, \cdot) \quad \text { depends continuously on } x \text { with values in } W(Z) .
\end{array}\right.
$$

Noting that $A(x, z) \equiv\left(\alpha_{i j}\left(x, z_{1}\right)\right)$ does not depend on $z_{2}, \ldots, z_{n}$, we may let $\varphi^{k}(\cdot, \cdot)$ be independent of $z_{2}, \ldots, z_{n}$. Then (2.10) implies

$$
e_{1}^{T}\left[A(x, z)\left(\varphi_{z_{1}}^{k}\left(x, z_{1}\right) e_{1}-e_{k}\right)\right]_{z_{1}}=0,
$$

which leads to

$$
e_{1}^{T} A(x, z) e_{1} \varphi_{z_{1}}^{k}\left(x, z_{1}\right)-e_{1}^{T} A(x, z) e_{k}=\rho^{k}(x),
$$

with $\rho^{k}(\cdot)$ being undetermined. Hence,

$$
\varphi_{z_{1}}^{k}\left(x, z_{1}\right)=\frac{\rho^{k}(x)+\alpha_{1 k}\left(x, z_{1}\right)}{\alpha_{11}\left(x, z_{1}\right)} .
$$

Consequently,

$$
\varphi^{k}\left(x, z_{1}\right)=\varphi^{k}(x, 0)+\int_{0}^{z_{1}} \frac{\rho^{k}(x)+\alpha_{1 k}\left(x, \zeta_{1}\right)}{\alpha_{11}\left(x, \zeta_{1}\right)} d \zeta_{1}
$$

To meet the periodic condition $\varphi^{k}\left(x, z_{1}+1\right)=\varphi^{k}\left(x, z_{1}\right)$, we require

$$
\int_{0}^{1} \frac{\rho^{k}(x)+\alpha_{1 k}\left(x, \zeta_{1}\right)}{\alpha_{11}\left(x, \zeta_{1}\right)} d \zeta_{1}=0
$$

Thus,

$$
\rho^{k}(x)=-\left[\int_{0}^{1} \frac{d \zeta_{1}}{\alpha_{11}\left(x, \zeta_{1}\right)}\right]^{-1} \int_{0}^{1} \frac{\alpha_{1 k}\left(x, \zeta_{1}\right)}{\alpha_{11}\left(x, \zeta_{1}\right)} d \zeta_{1}
$$

By (2.2), we have

$$
\int_{0}^{1} \frac{d \zeta_{1}}{\alpha_{11}\left(x, \zeta_{1}\right)}=\frac{\delta}{b_{11}(x)}+\frac{1-\delta}{c_{11}(x)}
$$

and

$$
\int_{0}^{1} \frac{\alpha_{1 k}\left(x, \zeta_{1}\right)}{\alpha_{11}\left(x, \zeta_{1}\right)} d \zeta_{1}=\frac{\delta b_{1 k}(x)}{b_{11}(x)}+\frac{(1-\delta) c_{1 k}(x)}{c_{11}(x)}
$$

Hence,

$$
\rho^{k}(x)=-\left[\frac{\delta}{b_{11}(x)}+\frac{1-\delta}{c_{11}(x)}\right]^{-1}\left[\frac{\delta b_{1 k}(x)}{b_{11}(x)}+\frac{(1-\delta) c_{1 k}(x)}{c_{11}(x)}\right]
$$

Copyright $@$ by SIAM. Unauthorized reproduction of this article is prohibited. 
Then

$$
\begin{aligned}
& \begin{array}{l}
q_{i j}(x)=\int_{0}^{1}\left[\alpha_{i j}\left(x, z_{1}\right)+\frac{\left[\rho^{i}(x)+\alpha_{1 i}\left(x, z_{1}\right)\right]\left[\rho^{j}(x)+\alpha_{1 j}\left(x, z_{1}\right)\right]}{\alpha_{11}\left(x, z_{1}\right)}\right. \\
\left.\quad-\alpha_{1 j}\left(x, z_{1}\right) \frac{\rho^{i}(x)+\alpha_{1 i}\left(x, z_{1}\right)}{\alpha_{11}\left(x, z_{1}\right)}-\alpha_{1 i}\left(x, z_{1}\right) \frac{\rho^{j}(x)+\alpha_{1 j}\left(x, z_{1}\right)}{\alpha_{11}\left(x, z_{1}\right)}\right] d z_{1} \\
=\int_{0}^{1}\left[\alpha_{i j}\left(x, z_{1}\right)+\frac{\rho^{i}(x) \rho^{j}(x)-\alpha_{1 i}\left(x, z_{1}\right) \alpha_{1 j}\left(x, z_{1}\right)}{\alpha_{11}\left(x, z_{1}\right)}\right] d z_{1} \\
=\delta b_{i j}(x)+(1-\delta) c_{i j}(x)+\delta \frac{\rho^{i}(x) \rho^{j}(x)-b_{1 i}(x) b_{1 j}(x)}{b_{11}(x)} \\
\quad+(1-\delta) \frac{\rho^{i}(x) \rho^{j}(x)-c_{1 i}(x) c_{1 j}(x)}{c_{11}(x)} \\
=\delta b_{i j}(x)+(1-\delta) c_{i j}(x)-\frac{\delta}{b_{11}(x)} b_{1 i}(x) b_{1 j}(x)-\frac{1-\delta}{c_{11}(x)} c_{1 j}(x) c_{1 j}(x) \\
\quad+\left[\frac{\delta}{b_{11}(x)}+\frac{1-\delta}{c_{11}(x)}\right]^{-1}\left[\frac{\delta b_{1 i}(x)}{b_{11}(x)}+\frac{(1-\delta) c_{1 i}(x)}{c_{11}(x)}\right]\left[\frac{\delta b_{1 j}(x)}{b_{11}(x)}+\frac{(1-\delta) c_{1 j}(x)}{c_{11}(x)}\right] \\
=\delta b_{i j}(x)+(1-\delta) c_{i j}(x) \\
\quad+\frac{\delta(1-\delta)\left[b_{1 i}(x) c_{1 j}(x)+b_{1 j}(x) c_{1 j}(x)-b_{1 i}(x) b_{1 j}(x)-c_{1 i}(x) c_{1 j}(x)\right]}{(1-\delta) b_{11}(x)+\delta c_{11}(x)} \\
=\delta b_{i j}(x)+(1-\delta) c_{i j}(x)-\frac{\delta(1-\delta)\left[b_{1 i}(x)-c_{1 i}(x)\right]\left[b_{1 j}(x)-c_{1 j}(x)\right]}{(1-\delta) b_{11}(x)+\delta c_{11}(x)}
\end{array}
\end{aligned}
$$

proving our conclusion.

Note that if $S$ is a positive definite matrix with its eigenvalues on $[\lambda, \Lambda](\Lambda>\lambda>$ $0)$, then $I-\lambda S^{-1}$ is a positive semidefinite matrix with its eigenvalues on $\left[0,1-\frac{\lambda}{\Lambda}\right]$. Therefore

$$
\begin{gathered}
\left|\left\langle\left(S-\lambda I_{n}\right) \xi, \eta\right\rangle\right|^{2}=\left|\left\langle\left(I_{n}-\lambda S^{-1}\right) S^{\frac{1}{2}} \xi, S^{\frac{1}{2}} \eta\right\rangle\right|^{2} \\
\leq\left(1-\frac{\lambda}{\Lambda}\right)^{2}\langle S \xi, \xi\rangle\langle S \eta, \eta\rangle \leq\left(1-\frac{\lambda}{\Lambda}\right)\langle S \xi, \xi\rangle\langle S \eta, \eta\rangle .
\end{gathered}
$$

Consequently, if $e_{i} \in \mathbb{R}^{n}$ is the vector where the $i$ th coordinate is 1 and all others are zero, then for any $\xi \in \mathbb{R}^{n}$,

$$
\begin{aligned}
& \langle Q(x) \xi, \xi\rangle=\sum_{i, j=1}^{n} q_{i j}(x) \xi_{i} \xi_{j} \\
& =\delta\langle B(x) \xi, \xi\rangle+(1-\delta)\langle C(x) \xi, \xi\rangle-\frac{\delta(1-\delta)\left(\left\langle\left[B(x)-I_{n}\right] \xi, e_{1}\right\rangle+\left\langle\left[I_{n}-C(x)\right] \xi, e_{1}\right\rangle\right)^{2}}{(1-\delta)\left\langle B(x) e_{1}, e_{1}\right\rangle+\delta\left\langle C(x) e_{1}, e_{1}\right\rangle} \\
& \geq \delta\langle B(x) \xi, \xi\rangle+(1-\delta)\langle C(x) \xi, \xi\rangle \\
& \quad-\delta(1-\delta)\left(1-\frac{\lambda}{\Lambda}\right) \frac{\left(\sqrt{\langle B(x) \xi, \xi\rangle\left\langle B(x) e_{1}, e_{1}\right\rangle}+\sqrt{\langle C(x) \xi, \xi\rangle\left\langle C(x) e_{1}, e_{1}\right\rangle}\right)^{2}}{(1-\delta)\left\langle B(x) e_{1}, e_{1}\right\rangle+\delta\left\langle C(x) e_{1}, e_{1}\right\rangle} \\
& \geq \delta(1-\delta) \frac{\lambda}{\Lambda} \frac{\left(\sqrt{\langle B(x) \xi, \xi\rangle\left\langle B(x) e_{1}, e_{1}\right\rangle}+\sqrt{\langle C(x) \xi, \xi\rangle\left\langle C(x) e_{1}, e_{1}\right\rangle}\right)^{2}}{(1-\delta)\left\langle B(x) e_{1}, e_{1}\right\rangle+\delta\left\langle C(x) e_{1}, e_{1}\right\rangle} \\
& \geq \frac{4 \delta(1-\delta) \lambda^{3}|\xi|^{2}}{\Lambda^{2}} .
\end{aligned}
$$

Copyright (c) by SIAM. Unauthorized reproduction of this article is prohibited. 
Also, of course,

$$
\begin{aligned}
& \langle Q(x) \xi, \xi\rangle=\sum_{i, j=1}^{n} q_{i j}(x) \xi_{i} \xi_{j} \\
& =\delta\langle B(x) \xi, \xi\rangle+(1-\delta)\langle C(x) \xi, \xi\rangle-\frac{\delta(1-\delta)\left\langle[B(x)-C(x)] \xi, e_{1}\right\rangle\left\langle[B(x)-C(x)] \xi, e_{1}\right\rangle}{(1-\delta)\left\langle B(x) e_{1}, e_{1}\right\rangle+\delta\left\langle C(x) e_{1}, e_{1}\right\rangle} \\
& \leq \Lambda|\xi|^{2}+\frac{\delta(1-\delta) 4 \Lambda^{2}|\xi|^{2}}{\lambda} \leq \frac{2 \Lambda^{2}}{\lambda}|\xi|^{2} .
\end{aligned}
$$

Thus, we verified directly that $Q(\cdot) \in L^{\infty}\left(\Omega ; \mathcal{S}_{+}^{n}\right)$, and (2.6) is uniformly elliptic.

The following result is concerned with the well-posedness and regularity of state equation (1.1).

Lemma 2.2. Let (S1)-(S4) hold. Then for any $u(\cdot) \in \mathcal{U}$, (1.1) admits a unique weak solution $y(\cdot) \in H_{0}^{1}(\Omega) \cap L^{\infty}(\Omega)$. Furthermore, there exists a constant $K>0$, independent of $u(\cdot) \in \mathcal{U}$, such that

$$
\|y(\cdot)\|_{H_{0}^{1}(\Omega)}+\|y(\cdot)\|_{L^{\infty}(\Omega)} \leq K .
$$

The existence of a weak solution to $(1.1)$ in $H_{0}^{1}(\Omega)$ together with the $H_{0}^{1}(\Omega)$-norm estimate follows easily from the variational structure of (1.1), while the uniqueness of the weak solution follows from (1.4) and (1.5). Finally, the boundedness of the weak solution in $L^{\infty}(\Omega)$ follows from (1.4)-(1.6) and the standard De Giorgi iteration (note that by (1.5),

$$
f(x, y(x), u(x))-f(x, 0, u(x))=\left[\int_{0}^{1} f_{y}(x, \beta y(x), u(x)) d \beta\right] y(x)
$$

is a good term in De Giorgi iteration).

Lemma 2.3. Let $n \geq 2$. Let $\xi, \eta \in \mathbb{R}^{n}$ be two nonzero vectors. Then

$$
\sup _{|x|=1} x^{\top}\left(\xi \eta^{\top}+\eta \xi^{\top}\right) x=|\xi||\eta|+\xi^{\top} \eta .
$$

Proof. First of all, we may write (2.19) as

$$
\sup _{|x|=1} x^{\top}\left(\frac{\xi}{|\xi|} \frac{\eta^{\top}}{|\eta|}+\frac{\eta}{|\eta|} \frac{\xi^{\top}}{|\xi|}\right) x=1+\frac{\xi^{\top}}{|\xi|} \frac{\eta}{|\eta|} .
$$

Thus, without loss of generality, we may assume that $|\xi|=|\eta|=1$. Since $M \triangleq \xi \eta^{\top}+$ $\eta \xi^{\top}$ is a symmetric matrix, the spectrum $\sigma(M)$ of $M$ is contained in $\mathbb{R}$. Moreover, the left-hand side of (2.19) is the maximum eigenvalue of $M$. Hence, we need only to calculate the eigenvalues of $M$. To this end, we may further assume that $\xi=e_{1}$ (which can be achieved by making an orthogonal transformation) and $\eta=\left(\eta_{1}, \widehat{\eta}\right)$ with $\widehat{\eta} \in \mathbb{R}^{n-1}$, and

$$
|\eta|^{2}=\left|\eta_{1}\right|^{2}+|\widehat{\eta}|^{2}=1
$$

Then

$$
M=\xi \eta^{\top}+\eta \xi^{\top}=\left(\begin{array}{cc}
2 \eta_{1} & \widehat{\eta}^{\top} \\
\widehat{\eta} & 0
\end{array}\right)
$$

Copyright (c) by SIAM. Unauthorized reproduction of this article is prohibited. 
Thus, its characteristic equation reads

$$
0=\left|\begin{array}{cc}
\lambda-2 \eta_{1} & -\widehat{\eta}^{\top} \\
-\widehat{\eta} & \lambda I_{n-1}
\end{array}\right|=\lambda^{n-2}\left(\lambda^{2}-2 \eta_{1}-1+\eta_{1}^{2}\right)=\lambda^{n-2}\left[\left(\lambda-\eta_{1}\right)^{2}-1\right] .
$$

Hence, in this case,

$$
\max \sigma(M)=1+\eta_{1}=1+e_{1}^{\top} \eta=1+\xi^{\top} \eta .
$$

This proves the lemma.

3. Proof of the main theorem. In this section, we present a proof of our main theorem. The proof is divided into several steps. Let $\bar{u}(\cdot) \in \mathcal{U}$ be an optimal control and let $\bar{y}(\cdot)$ be the corresponding optimal state. Let $u(\cdot) \in \mathcal{U}$ be fixed.

I. Homogenizing uniform spike variation for the control. Let $\delta \in(0,1)$ and $\varepsilon>0$. For any $x=\left(x_{1}, x_{2}, \ldots, x_{n}\right) \in \Omega$, define

$$
u^{\delta, \varepsilon}(x)=\left\{\begin{array}{l}
u(x) \quad \text { if } \frac{x_{1}}{\varepsilon} \in \bigcup_{k=-\infty}^{+\infty}[k, k+\delta], \\
\bar{u}(x) \quad \text { if } \frac{x_{1}}{\varepsilon} \in \bigcup_{k=-\infty}^{+\infty}(k+\delta, k+1) .
\end{array}\right.
$$

We refer to $u^{\delta, \varepsilon}(\cdot)$ as a uniform spike variation of the control $\bar{u}(\cdot)$ in the direction of $u(\cdot)$, which is uniformly highly oscillatory and will be homogenized. Let $y^{\delta, \varepsilon}(\cdot)$ be the state corresponding to $u^{\delta, \varepsilon}(\cdot)$. Then

$$
\left\{\begin{array}{l}
-\nabla \cdot\left(A^{\delta, \varepsilon}(x) \nabla y^{\delta, \varepsilon}(x)\right)=f\left(x, y^{\delta, \varepsilon}(x), u^{\delta, \varepsilon}(x)\right) \quad \text { in } \Omega \\
\left.y^{\delta, \varepsilon}\right|_{\partial \Omega}=0
\end{array}\right.
$$

where

$$
A^{\delta, \varepsilon}(x)=A(x, \bar{u}(x))+g\left(\frac{x_{1}}{\varepsilon}\right)(A(x, u(x))-A(x, \bar{u}(x)))
$$

and $g(\cdot)$ is the function defined by $(2.2)$. By Lemma 2.2, there exists a constant $K>0$, independent of $\delta, \varepsilon$, such that

$$
\left\|y^{\delta, \varepsilon}(\cdot)\right\|_{H_{0}^{1}(\Omega)}+\left\|y^{\delta, \varepsilon}(\cdot)\right\|_{L^{\infty}(\Omega)} \leq K .
$$

Consequently, by (1.6),

$$
\left|f\left(x, y^{\delta, \varepsilon}(x), u^{\delta, \varepsilon}(x)\right)\right| \leq M_{K} .
$$

By (3.2), for fixed $\delta \in(0,1)$, we can extract a sequence (still denoted by itself) such that $y^{\delta, \varepsilon}(\cdot)$ converges to a function $y^{\delta}(\cdot)$ weakly in $H_{0}^{1}(\Omega)$, strongly in $L^{2}(\Omega)$, and almost everywhere in $\Omega$ as $\varepsilon \rightarrow 0^{+}$.

By (1.6) and (3.2),

$$
\left|f\left(x, y^{\delta, \varepsilon}(x), u^{\delta, \varepsilon}(x)\right)-f\left(x, y^{\delta}(x), u^{\delta, \varepsilon}(x)\right)\right| \leq M_{K}\left|y^{\delta, \varepsilon}(x)-y^{\delta}(x)\right| .
$$

Copyright $@$ by SIAM. Unauthorized reproduction of this article is prohibited. 
On the other hand, using a generalization of the Riemann-Lebesgue theorem (see [13, Chapter II, Theorem 4.15]), it is not very hard to prove that for any $h \in L^{2}(\Omega)$, when $\varepsilon \rightarrow 0^{+}$,

$$
\begin{aligned}
& \int_{\Omega} f\left(x, y^{\delta}(x), u^{\delta, \varepsilon}(x)\right) h(x) d x \\
& =\int_{\Omega} g\left(\frac{x_{1}}{\varepsilon}\right) f\left(x, y^{\delta}(x), \bar{u}(x)\right) h(x) d x+\int_{\Omega}\left(1-g\left(\frac{x_{1}}{\varepsilon}\right)\right) f\left(x, y^{\delta}(x), u(x)\right) h(x) d x \\
& \rightarrow \int_{0}^{1} g(t) d t \int_{\Omega} f\left(x, y^{\delta}(x), \bar{u}(x)\right) h(x) d x+\int_{0}^{1}(1-g(t)) d t \int_{\Omega} f\left(x, y^{\delta}(x), u(x)\right) h(x) d x \\
& =(1-\delta) \int_{\Omega} f\left(x, y^{\delta}(x), \bar{u}(x)\right) h(x) d x+\delta \int_{\Omega} f\left(x, y^{\delta}(x), u(x)\right) h(x) d x .
\end{aligned}
$$

Combining the above with (3.4), we get that along a subsequence $\varepsilon \rightarrow 0^{+}$,

$$
\begin{array}{r}
f\left(x, y^{\delta, \varepsilon}(x), u^{\delta, \varepsilon}(x)\right) \rightarrow \delta f\left(x, y^{\delta}(x), u(x)\right)+(1-\delta) f\left(x, y^{\delta}(x), \bar{u}(x)\right), \\
\text { weakly in } L^{2}(\Omega) .
\end{array}
$$

Let

$$
h^{\delta, \varepsilon}(x)=f\left(x, y^{\delta, \varepsilon}(x), u^{\delta, \varepsilon}(x)\right)-\left(\delta f\left(x, y^{\delta}(x), u(x)\right)+(1-\delta) f\left(x, y^{\delta}(x), \bar{u}(x)\right)\right),
$$

and let $z^{\delta, \varepsilon}(\cdot)$ be the solution of

$$
\left\{\begin{array}{l}
-\nabla \cdot\left(A^{\delta, \varepsilon}(x) \nabla z^{\delta, \varepsilon}(x)\right)=h^{\delta, \varepsilon}(x) \text { in } \Omega \\
\left.z^{\delta, \varepsilon}\right|_{\partial \Omega}=0
\end{array}\right.
$$

Then

$$
\begin{aligned}
\lambda \int_{\Omega}\left|\nabla z^{\delta, \varepsilon}(x)\right|^{2} & \leq \int_{\Omega}\left\langle A^{\delta, e}(x) \nabla z^{\delta, \varepsilon}(x), \nabla z^{\delta, \varepsilon}(x)\right\rangle d x \\
& =\int_{\Omega} z^{\delta, \varepsilon}(x) h^{\delta, \varepsilon}(x) d x
\end{aligned}
$$

Consequently, by (3.5), along a subsequence $\varepsilon \rightarrow 0^{+}$, one has

$$
z^{\delta, \varepsilon}(\cdot) \rightarrow 0, \quad \text { strongly in } H_{0}^{1}(\Omega)
$$

By Proposition 2.1,

$$
\left(y^{\delta, \varepsilon}(\cdot)-z^{\delta, \varepsilon}(\cdot)\right) \rightarrow y^{\delta}(\cdot), \quad \text { weakly in } H_{0}^{1}(\Omega),
$$

where $y^{\delta}(\cdot)$ is the weak solution of

$$
\left\{\begin{aligned}
-\nabla \cdot\left(Q^{\delta}(x) \nabla y^{\delta}(x)\right)= & \delta f\left(x, y^{\delta}(x), u(x)\right) \\
& +(1-\delta) f\left(x, y^{\delta}(x), \bar{u}(x)\right) \quad \text { in } \Omega \\
\left.y^{\delta}\right|_{\partial \Omega}=0, &
\end{aligned}\right.
$$

and $Q^{\delta}(\cdot)=\left(q_{i j}^{\delta}(\cdot)\right)$ with

$$
\begin{aligned}
& q_{i j}^{\delta}(x)= \delta a_{i j}(x, u(x))+(1-\delta) a_{i j}(x, \bar{u}(x)) \\
&-\frac{\delta(1-\delta)\left[a_{1 i}(x, u(x))-a_{1 i}(x, \bar{u}(x))\right]\left[a_{1 j}(x, u(x))-a_{1 j}(x, \bar{u}(x))\right]}{(1-\delta) a_{11}(x, u(x))+\delta a_{11}(x, \bar{u}(x))}, \\
& 1 \leq i, j \leq n .
\end{aligned}
$$

Copyright (C) by SIAM. Unauthorized reproduction of this article is prohibited. 
Combining (3.10) with (3.9), along a subsequence, we obtain

$$
y^{\delta, \varepsilon}(\cdot) \rightarrow y^{\delta}(\cdot), \quad \text { weakly in } H_{0}^{1}(\Omega) .
$$

Note that for fixed $\delta \in(0,1)$, since any subsequence of $y^{\delta, \varepsilon}(\cdot)$ has a further subsequence converging to the same $y^{\delta}(\cdot)$ weakly in $H_{0}^{1}(\Omega), y^{\delta, \varepsilon}(\cdot)$ itself must converge to $y^{\delta}(\cdot)$ weakly in $H_{0}^{1}(\Omega)$. We call (3.11) an intermediate variational equation associated with the optimal pair $(\bar{y}(\cdot), \bar{u}(\cdot))$, with the parameter $\delta \in(0,1)$. We observe that the dependence of $(3.11)$ on the parameter $\delta$, through the expression $q_{i j}^{\delta}(\cdot)$ and the righthand side, is explicit, which is crucial in further taking the limit as $\delta \rightarrow 0$. This is exactly the main advantage of introducing the homogenized spike variation of the control.

In addition, by the optimality of $\bar{u}(\cdot)$, we have

$$
\begin{aligned}
J(\bar{u}(\cdot)) & \leq \lim _{\varepsilon \rightarrow 0^{+}} J\left(u^{\delta, \varepsilon}(\cdot)\right) \\
& =\delta \int_{\Omega} f^{0}\left(x, y^{\delta}(x), u(x)\right) d x+(1-\delta) \int_{\Omega} f^{0}\left(x, y^{\delta}(x), \bar{u}(x)\right) d x .
\end{aligned}
$$

II. Linearized state equation. We now would like to let $\delta \rightarrow 0$. Denote

$$
Y^{\delta}(x)=\frac{y^{\delta}(x)-\bar{y}(x)}{\delta}, \quad x \in \Omega .
$$

Then it follows from (3.11) that

$$
\left\{\begin{aligned}
-\nabla \cdot\left(A(x, \bar{u}(x)) \nabla Y^{\delta}(x)\right)=\left[\int_{0}^{1} f_{y}\left(x, \bar{y}(x)+t\left(y^{\delta}(x)-\bar{y}(x)\right), \bar{u}(x)\right) d t\right] Y^{\delta}(x) \\
+\nabla \cdot\left(\frac{Q^{\delta}(x)-A(x, \bar{u}(x))}{\delta} \nabla y^{\delta}(x)\right) \\
\quad+f\left(x, y^{\delta}(x), u(x)\right)-f\left(x, y^{\delta}(x), \bar{u}(x)\right) \text { in } \Omega \\
\left.Y^{\delta}\right|_{\partial \Omega}=0 .
\end{aligned}\right.
$$

Noting that as $\delta \rightarrow 0$,

$$
\begin{aligned}
& \frac{q_{i j}^{\delta}(x)-a_{i j}(x, \bar{u}(x))}{\delta}=a_{i j}(x, u(x))-a_{i j}(x, \bar{u}(x)) \\
& -\frac{(1-\delta)\left[a_{1 i}(x, u(x))-a_{1 i}(x, \bar{u}(x))\right]\left[a_{1 j}(x, u(x))-a_{1 j}(x, \bar{u}(x))\right]}{(1-\delta) a_{11}(x, u(x))+\delta a_{11}(x, \bar{u}(x))}
\end{aligned}
$$

converges in $L^{\infty}(\Omega)$ to

$$
\begin{aligned}
& \theta_{i j}(x)= a_{i j}(x, u(x))-a_{i j}(x, \bar{u}(x)) \\
&-\frac{\left[a_{1 i}(x, u(x))-a_{1 i}(x, \bar{u}(x))\right]\left[a_{1 j}(x, u(x))-a_{1 j}(x, \bar{u}(x))\right]}{a_{11}(x, u(x))}, \\
& 1 \leq i, j \leq n .
\end{aligned}
$$

On the other hand, $y^{\delta}(\cdot)$ is bounded uniformly in $H_{0}^{1}(\Omega)$. Thus, we can prove step by step that $Y^{\delta}(\cdot)$ is bounded uniformly in $H_{0}^{1}(\Omega), y^{\delta}(\cdot)$ converges to $\bar{y}(\cdot)$ strongly 
in $H_{0}^{1}(\Omega)$, and $Y^{\delta}(\cdot)$ converges to $Y(\cdot)$ weakly in $H_{0}^{1}(\Omega)$, with $Y(\cdot)$ being the weak solution of

$$
\left\{\begin{array}{c}
-\nabla \cdot(A(x, \bar{u}(x)) \nabla Y(x))=f_{y}(x, \bar{y}(x), \bar{u}(x)) Y(x)+\nabla \cdot(\Theta(x) \nabla \bar{y}(x)) \\
+f(x, \bar{y}(x), u(x))-f(x, \bar{y}(x), \bar{u}(x)), \\
\text { in } \Omega
\end{array}\right.
$$

where

$$
\begin{aligned}
\Theta(x)= & \left(\theta_{i j}(x)\right)=A(x, u(x))-A(x, \bar{u}(x)) \\
& -\frac{[A(x, u(x))-A(x, \bar{u}(x))] e_{1} e_{1}^{\top}[A(x, u(x))-A(x, \bar{u}(x))]}{e_{1}^{\top} A(x, u(x)) e_{1}} .
\end{aligned}
$$

From (3.14), we also have

$$
\begin{aligned}
0 \leq \lim _{\delta \rightarrow 0^{+}} & {\left[\int_{\Omega}\left(f^{0}\left(x, y^{\delta}(x), u(x)\right)-f^{0}\left(x, y^{\delta}(x), \bar{u}(x)\right)\right) d x\right.} \\
& \left.+\int_{\Omega} d x \int_{0}^{1} f_{y}^{0}\left(x, \bar{y}(x)+\beta\left(y^{\delta}(x)-\bar{y}(x)\right), \bar{u}(x)\right) Y^{\delta}(x) d \beta\right] \\
= & \int_{\Omega}\left(f^{0}(x, \bar{y}(x), u(x))-f^{0}(x, \bar{y}(x), \bar{u}(x))+f_{y}^{0}(x, \bar{y}(x), \bar{u}(x)) Y(x)\right) d x .
\end{aligned}
$$

III. Duality. Let $\bar{\psi}(\cdot)$ be the solution of the adjoint equation (1.8). Then (3.17) becomes

$$
\begin{aligned}
& 0 \leq \int_{\Omega}\left(f^{0}(x, \bar{y}(x), u(x))-f^{0}(x, \bar{y}(x), \bar{u}(x))\right) d x \\
& +\int_{\Omega}\left[\nabla \cdot(A(x, \bar{u}(x)) \nabla \bar{\psi}(x))+f_{y}(x, \bar{y}(x), \bar{u}(x)) \bar{\psi}(x)\right] Y(x) d x \\
& =\int_{\Omega}\left(f^{0}(x, \bar{y}(x), u(x))-f^{0}(x, \bar{y}(x), \bar{u}(x))\right) d x \\
& +\int_{\Omega}\left[\nabla \cdot(A(x, \bar{u}(x)) \nabla Y(x))+f_{y}(x, \bar{y}(x), \bar{u}(x)) Y(x)\right] \bar{\psi}(x) d x \\
& =\int_{\Omega}\left(f^{0}(x, \bar{y}(x), u(x))-f^{0}(x, \bar{y}(x), \bar{u}(x))\right) d x \\
& -\int_{\Omega}(f(x, \bar{y}(x), u(x))-f(x, \bar{y}(x), \bar{u}(x))) \bar{\psi}(x) d x \\
& +\int_{\Omega}\langle\Theta(x) \nabla \bar{y}(x), \nabla \bar{\psi}(x)\rangle d x \\
& =\int_{\Omega}\left[\left(f(x, \bar{y}(x), \bar{u}(x)) \bar{\psi}(x)-f^{0}(x, \bar{y}(x), \bar{u}(x))-\langle A(x, \bar{u}(x)) \nabla \bar{y}(x), \nabla \bar{\psi}(x)\rangle\right)\right. \\
& \left.-\left(f(x, \bar{y}(x), u(x)) \bar{\psi}(x)-f^{0}(x, \bar{y}(x), u(x))-\langle A(x, u(x)) \nabla \bar{y}(x), \nabla \bar{\psi}(x)\rangle\right)\right] d x \\
& -\int_{\Omega} \frac{e_{1}^{\top}[A(x, u(x))-A(x, \bar{u}(x))] \nabla \bar{y}(x) \nabla \bar{\psi}(x)^{\top}[A(x, u(x))-A(x, \bar{u}(x))] e_{1}}{2 e_{1}^{\top} A(x, u(x)) e_{1}} d x \\
& =\int_{\Omega}(H(x, \bar{y}(x), \bar{\psi}(x), \nabla \bar{y}(x), \nabla \bar{\psi}(x), \bar{u}(x))-H(x, \bar{y}(x), \bar{\psi}(x), \nabla \bar{y}(x), \nabla \bar{\psi}(x), u(x))) d x \\
& -\int_{\Omega} \Phi\left(A(x, u)-A(x, \bar{u}), A(x, u), \nabla \bar{y}, \nabla \bar{\psi}, e_{1}\right) d x,
\end{aligned}
$$

Copyright $@$ ( ) by SIAM. Unauthorized reproduction of this article is prohibited. 
where $H$ is defined by (1.11), and

$$
\Phi(A, B, \xi, \eta, \nu)=\frac{\nu^{\top} A \xi \eta^{\top} A \nu}{2 \nu^{\top} B \nu}, \quad(A, B, \xi, \eta, \nu) \in \mathcal{S}^{n} \times \mathcal{S}_{+}^{n} \times \mathbb{R}^{n} \times \mathbb{R}^{n} \times \mathbb{R}^{n},
$$

where $\mathcal{S}^{n}$ is the set of all $(n \times n)$ real symmetric matrices.

IV. Maximum condition. By a standard argument [8], we have from (3.21) that

$$
\begin{gathered}
H(x, \bar{y}(x), \bar{\psi}(x), \nabla \bar{y}(x), \nabla \bar{\psi}(x), \bar{u}(x))-H(x, \bar{y}(x), \bar{\psi}(x), \nabla \bar{y}(x), \nabla \bar{\psi}(x), v) \\
\geq \Phi\left(A(x, v)-A(x, \bar{u}), A(x, v), \nabla \bar{y}(x), \nabla \bar{\psi}(x), e_{1}\right) \\
\forall v \in U, \quad \text { a.e. } x \in \Omega .
\end{gathered}
$$

If $n=1$, the above gives (1.9).

If $n \geq 2$, we can generalize (3.23) to the following:

$$
\begin{gathered}
H(x, \bar{y}(x), \bar{\psi}(x), \nabla \bar{y}(x), \nabla \bar{\psi}(x), \bar{u}(x))-H(x, \bar{y}(x), \bar{\psi}(x), \nabla \bar{y}(x), \nabla \bar{\psi}(x), v) \\
\geq \Phi(A(x, v)-A(x, \bar{u}), A(x, v), \nabla \bar{y}(x), \nabla \bar{\psi}(x), e) \\
\forall v \in U, e \in S^{n-1}, \quad \text { a.e. } x \in \Omega,
\end{gathered}
$$

where $S^{n-1}=\left\{x \in \mathbb{R}^{n}|| x \mid=1\right\}$. Now, given $(x, v)$, we denote

$$
\left\{\begin{array}{l}
\mu=A(x, v)^{\frac{1}{2}} e, \\
\xi=A(x, v)^{-\frac{1}{2}}[A(x, v)-A(x, \bar{u}(x))] \nabla \bar{y}(x), \\
\eta=A(x, v)^{-\frac{1}{2}}[A(x, v)-A(x, \bar{u}(x))] \nabla \bar{\psi}(x) .
\end{array}\right.
$$

Then the above becomes

$$
\begin{aligned}
H(x, \bar{y}(x), \bar{\psi}(x), \nabla \bar{y}(x), \nabla \bar{\psi}(x), \bar{u}(x))-H(x, \bar{y}(x), \bar{\psi}(x), \nabla \bar{y}(x), \nabla \bar{\psi}(x), v) \\
\geq \frac{\mu^{\top} \xi \eta^{\top} \mu}{|\mu|^{2}} .
\end{aligned}
$$

When $e$ runs over $S^{n-1}, \mu=\frac{A(x, v)^{-\frac{1}{2}} e}{\left|A(x, v)^{-\frac{1}{2}} e\right|}$ will run over $S^{n-1}$. Hence, by Lemma 2.3,

$$
\sup _{\mu \neq 0} \frac{\mu^{\top} \xi \eta^{\top} \mu}{|\mu|^{2}}=\frac{|\xi||\eta|+\xi^{\top} \eta}{2} .
$$

Combining the above, we obtain (1.10). This completes the proof of Theorem 1.1.

4. Problem with state constraints. In this section, we will consider the cases of state constraint. We assume the following.

(S6) Let $\mathcal{Y}$ be a Banach space with strictly convex dual $\mathcal{Y}^{*}$, let $F: H_{0}^{1}(\Omega) \rightarrow \mathcal{Y}$ be continuous Fréchet differentiable, and let $E \subseteq \mathcal{Y}$ be closed and convex.

As in Chapter 5 of [8], many state constraints can be stated in the following type:

$$
F(y(\cdot)) \in E .
$$

Let $\mathcal{P}_{a d}$ be the set of all pairs $(y(\cdot), u(\cdot))$ satisfying (1.1) and (4.1). Any $(y(\cdot), u(\cdot)) \in$ $\mathcal{P}_{a d}$ is called an admissible pair. The set $\mathcal{U}_{a d} \equiv\left\{u(\cdot) \in \mathcal{U} \mid(y(\cdot ; u(\cdot)), u(\cdot)) \in \mathcal{P}_{a d}\right\}$ is 
called the set of admissible controls. The following is our optimal control problem with state constraint.

Problem (SC). Find a control $\bar{u}(\cdot) \in \mathcal{U}_{a d}$ such that

$$
J(\bar{u}(\cdot))=\inf _{u(\cdot) \in \mathcal{U}} J(u(\cdot)) .
$$

To state necessary conditions for optimal controls of Problem (SC), we need to recall the notion of finite codimensionality and Ekeland's variational principle (see [8, Chapter 4], for example).

Definition 4.1. Let $X$ be a Banach space and let $X_{0}$ be a subspace of $X$. We say that $X_{0}$ is finite codimensional in $X$ if there exist $x_{1}, x_{2}, \ldots, x_{m} \in X$, such that

$$
\operatorname{span}\left\{X_{0}, x_{1}, \ldots, x_{n}\right\} \equiv \text { the space spanned by }\left\{X_{0}, x_{1}, \ldots, x_{n}\right\}=X .
$$

$A$ subset $S$ of $X$ is said to be finite codimensional in $X$ if for some $x_{0} \in S, \operatorname{span}(S-$ $\left.\left\{x_{0}\right\}\right) \equiv$ the closed subspace spanned by $\left\{x-x_{0} \mid x \in S\right\}$ is a finite codimensional subspace of $X$ and $\overline{\mathrm{co}} S \equiv$ the closed convex hull of $S-\left\{x_{0}\right\}$ has a nonempty interior in this subspace.

LEMma 4.2 (Ekeland's variational principle). Let $(V, d)$ be a complete metric space and let $F: V \rightarrow \mathbb{R}$ be a lower semicontinuous function bounded from below. Let $\delta>0$ and $v_{0} \in V$ be such that

$$
F\left(v_{0}\right) \leq \inf _{v \in V} F(v)+\delta .
$$

Then for any $\delta>0$, there exists a $v_{\delta} \in V$ such that

$$
F\left(v_{\delta}\right) \leq F\left(v_{0}\right), \quad d\left(v_{\delta}, v_{0}\right) \leq \sqrt{\delta}
$$

and for all $v \in V$,

$$
F\left(v_{\delta}\right) \leq F(v)+\sqrt{\delta} d\left(v, v_{\delta}\right)
$$

Now, let $(\bar{y}(\cdot), \bar{u}(\cdot))$ be an optimal pair of Problem (SC). Let $z=z(\cdot ; u(\cdot)) \in H_{0}^{1}(\Omega)$ be the unique weak solution of the following equation linearized equation at $(\bar{y}(\cdot), \bar{u}(\cdot))$ :

$$
\left\{\begin{aligned}
-\nabla \cdot(A(x, \bar{u}(x)) \nabla z(x))= & f_{y}(x, \bar{y}(x), \bar{u}(x)) z(x)+f(x, \bar{y}(x), u(x)) \\
& -f(x, \bar{y}(x), \bar{u}(x)) \quad \text { in } \Omega \\
\left.z\right|_{\partial \Omega}=0, &
\end{aligned}\right.
$$

and define the reachable set of variational system (4.6) as

$$
\mathcal{R}=\{z(\cdot ; u(\cdot)) \mid u(\cdot) \in \mathcal{U}\} .
$$

Now, let us state the necessary conditions of an optimal control to Problem (SC) as follows.

TheOrem 4.3. Let $(\mathrm{S} 1)-(\mathrm{S} 6)$ hold. Let $(\bar{y}(\cdot), \bar{u}(\cdot)) \in \mathcal{P}_{\text {ad }}$ be an optimal pair of Problem (SC). Let

$$
F^{\prime}(\bar{y}(\cdot)) \mathcal{R}-E \equiv\left\{\xi-\eta \mid \xi \in F^{\prime}(y(\cdot)) \mathcal{R}, \eta \in E\right\}
$$

Copyright $@$ by SIAM. Unauthorized reproduction of this article is prohibited. 
be finite codimensional in $\mathcal{Y}$. Then there exists a triple $\left(\bar{\psi}_{0}, \bar{\psi}(\cdot), \bar{\varphi}(\cdot)\right) \in \mathbb{R} \times H_{0}^{1}(\Omega) \times$ $\mathcal{Y}^{*}$ satisfying

$$
\begin{gathered}
\left\{\begin{array}{l}
\bar{\psi}_{0} \leq 0, \\
\left(\bar{\psi}_{0}, \bar{\varphi}(\cdot)\right) \neq 0, \\
\left(\bar{\psi}_{0}, \bar{\psi}(\cdot)\right) \neq 0 \quad \text { if } F^{\prime}(\bar{y}(\cdot))^{*} \text { is injective, }
\end{array}\right. \\
\langle\bar{\varphi}(\cdot), \eta-F(\bar{y}(\cdot))\rangle \mathcal{Y}^{*}, \mathcal{Y} \leq 0 \quad \forall \eta \in E, \\
\left\{\begin{array}{c}
-\nabla \cdot(A(x, \bar{u}(x)) \nabla \bar{\psi}(x))=f_{y}(x, \bar{y}(x), \bar{u}(x))^{\top} \bar{\psi}(x)+\psi_{0} f_{y}^{0}(x, \bar{y}(x), \bar{u}(x)) \\
-F^{\prime}(\bar{y}(\cdot))^{*} \bar{\varphi} \quad \text { in } \Omega,
\end{array}\right. \\
\left.\bar{\psi}\right|_{\partial \Omega}=0,
\end{gathered}
$$

such that when $n=1$,

$$
\begin{aligned}
& H\left(x, \bar{y}(x), \bar{\psi}_{0}, \bar{\psi}(x), \bar{y}^{\prime}(x), \bar{\psi}^{\prime}(x), \bar{u}(x)\right)-H\left(x, \bar{y}(x), \bar{\psi}_{0}, \bar{\psi}(x), \bar{y}^{\prime}(x), \bar{\psi}^{\prime}(x), v\right) \\
& \geq \frac{[A(x, v)-A(x, \bar{u}(x))]^{2}}{A(x, v)} \bar{y}^{\prime}(x) \bar{\psi}^{\prime}(x) \quad \forall v \in U, \quad \text { a.e. } x \in \Omega ;
\end{aligned}
$$

and when $n \geq 2$,

$$
\begin{gathered}
H\left(x, \bar{y}(x), \bar{\psi}_{0}, \bar{\psi}(x), \nabla \bar{y}(x), \nabla \bar{\psi}(x), \bar{u}(x)\right)-H\left(x, \bar{y}(x), \bar{\psi}_{0}, \bar{\psi}(x), \nabla \bar{y}(x), \nabla \bar{\psi}(x), v\right) \\
\geq \frac{1}{2}\left|A(x, v)^{-\frac{1}{2}}(A(x, \bar{u}(x))-A(x, v)) \nabla \bar{y}(x)\right|\left|A(x, v)^{-\frac{1}{2}}(A(x, \bar{u}(x))-A(x, v)) \nabla \bar{\psi}(x)\right| \\
+\frac{1}{2}\left\langle A(x, v)^{-\frac{1}{2}}(A(x, \bar{u}(x))-A(x, v)) \nabla \bar{y}(x), A(x, v)^{-\frac{1}{2}}(A(x, \bar{u}(x))-A(x, v)) \nabla \bar{\psi}(x)\right\rangle \\
\forall v \in U, \quad \text { a.e. } x \in \Omega,
\end{gathered}
$$

where

$$
\begin{aligned}
H\left(x, y, \psi_{0}, \psi, \xi, \eta, v\right)= & \langle\psi, f(x, y, v)\rangle+\psi_{0} f^{0}(x, y, v)-\langle A(x, v) \xi, \eta\rangle, \\
& (x, y, \psi, \xi, \eta, v) \in \mathbb{R}^{n} \times \mathbb{R} \times \mathbb{R} \times \mathbb{R}^{n} \times \mathbb{R}^{n} \times U .
\end{aligned}
$$

In the above, (4.9) is called the transversality condition. The proof of Theorem 4.3 is a combination of the proof of Theorem 1.1 and that of Theorem 1.2 of Chapter 5 in [8]. We give only a sketch of it here.

First, let

$$
\rho(u(\cdot), \tilde{u}(\cdot))=|\{x \in \Omega \mid u(x) \neq \tilde{u}(x)\}|,
$$

where $|D|$ is the Lebesgue measure of $D \subseteq \Omega$. We can prove that $(\mathcal{U}, \rho)$ is a complete metric space. For any $\delta>0$, let

$$
J_{\delta}(u(\cdot))=\left\{\left[(J(u(\cdot))-J(\bar{u}(\cdot))+\delta)^{+}\right]^{2}+d_{E}^{2}(F(y(\cdot ; u(\cdot))))\right\}^{\frac{1}{2}},
$$

where $d_{E}(x) \equiv \inf _{\tilde{x} \in E}|x-\tilde{x}|$, and $\bar{u}(\cdot) \in \mathcal{U}_{a d}$ is an optimal control. Then

$$
J_{\delta}(u(\cdot))>0 \quad \forall \delta>0, u(\cdot) \in \mathcal{U}
$$

Copyright $@$ ㅇ by SIAM. Unauthorized reproduction of this article is prohibited. 
and it follows easily from $(\mathrm{S} 1)-(\mathrm{S} 5)$ that $J_{\delta}(u(\cdot))$ is continuous on $(\mathcal{U}, \rho)$ and

$$
J_{\delta}(\bar{u}(\cdot)) \leq \inf _{u(\cdot) \in \mathcal{U}} J(u(\cdot))+\delta .
$$

Second, using Ekeland's variational principle, we have some $\bar{u}_{\delta}(\cdot) \in \mathcal{U}$ such that

$$
\begin{gathered}
J_{\delta}\left(\bar{u}_{\delta}(\cdot)\right) \leq J_{\delta}(\bar{u}(\cdot)), \\
\rho\left(\bar{u}_{\delta}(\cdot), \bar{u}(\cdot)\right) \leq \sqrt{\delta},
\end{gathered}
$$

and

$$
J_{\delta}\left(\bar{u}_{\delta}(\cdot)\right) \leq J_{\delta}(u(\cdot))+\sqrt{\delta} \rho\left(u(\cdot), \bar{u}_{\delta}(\cdot)\right) \quad \forall u(\cdot) \in \mathcal{U} .
$$

Note that (4.14) means that $\bar{u}_{\delta}(\cdot)$ is a minimum of $u(\cdot) \mapsto J_{\delta}(u(\cdot))+\rho\left(u(\cdot), \bar{u}_{\delta}(\cdot)\right)$ on $\mathcal{U}$.

By a discussion similar to the proof of Theorem 1.1, we can get that, analogous to (3.16) and (3.17), for any fixed $u(\cdot) \in \mathcal{U}$,

$$
\left\{\begin{array}{c}
-\nabla \cdot\left(A\left(x, \bar{u}_{\delta}(x)\right) \nabla Y_{\delta}(x)\right)=f_{y}\left(x, \bar{y}_{\delta}(x), \bar{u}_{\delta}(x)\right) Y_{\delta}(x)+\nabla \cdot\left(\Theta_{\delta}(x) \nabla \bar{y}_{\delta}(x)\right) \\
+f\left(x, \bar{y}_{\delta}(x), u(x)\right)-f\left(x, \bar{y}_{\delta}(x), \bar{u}(x)\right)
\end{array}\right.
$$

and

$$
\begin{aligned}
-\sqrt{\delta}|\Omega| \leq & \frac{\left(J\left(\bar{u}_{\delta}(\cdot)\right)-J(\bar{u}(\cdot))+\delta\right)^{+}}{J_{\delta}\left(\bar{u}_{\delta}\right)} \int_{\Omega}\left(f^{0}\left(x, \bar{y}_{\delta}(x), u(x)\right)-f^{0}\left(x, \bar{y}_{\delta}(x), \bar{u}_{\delta}(x)\right)\right. \\
& \left.+f_{y}^{0}\left(x, \bar{y}_{\delta}(x), \bar{u}_{\delta}(x)\right) Y_{\delta}(x)\right) d x \\
& +\left\langle\frac{d_{E}\left(F\left(\bar{y}_{\delta}(\cdot)\right)\right) \xi_{\delta}}{J_{\delta}\left(\bar{u}_{\delta}(\cdot)\right)}, F^{\prime}\left(\bar{y}_{\delta}(\cdot)\right) Y_{\delta}\right\rangle_{\mathcal{Y}^{*}, \mathcal{Y}}
\end{aligned}
$$

where $\bar{y}_{\delta}(\cdot)=y\left(\cdot ; \bar{u}_{\delta}(\cdot)\right)$,

$$
\begin{gathered}
\Theta_{\delta}(x)=A(x, u(x))-A\left(x, \bar{u}_{\delta}(x)\right) \\
-\frac{\left[A(x, u(x))-A\left(x, \bar{u}_{\delta}(x)\right)\right] e_{1} e_{1}^{\top}\left[A(x, u(x))-A\left(x, \bar{u}_{\delta}(x)\right)\right]}{e_{1}^{\top} A(x, u(x)) e_{1}}, \\
\xi_{\delta}(\cdot)= \begin{cases}\nabla d_{E}\left(F\left(\bar{y}_{\delta}(\cdot)\right)\right) & \text { if } F\left(\bar{y}_{\delta}(\cdot)\right) \notin E, \\
0 & \text { if } F\left(\bar{y}_{\delta}(\cdot)\right) \in E,\end{cases}
\end{gathered}
$$

and $\nabla d_{E}(\cdot)$ denotes the subdifferential of $d_{E}(\cdot)$. Denote

$$
\psi_{0, \delta}=-\frac{\left(J\left(\bar{u}_{\delta}(\cdot)\right)-J(\bar{u}(\cdot))+\delta\right)^{+}}{J_{\delta}\left(\bar{u}_{\delta}(\cdot)\right)}, \quad \varphi_{\varepsilon}(\cdot)=\frac{d_{E}\left(F\left(\bar{y}_{\delta}(\cdot)\right)\right) \xi_{\delta}(\cdot)}{J_{\delta}\left(\bar{u}_{\delta}(\cdot)\right)}
$$

Then (4.16) becomes

$$
\begin{aligned}
-\sqrt{\delta}|\Omega| \leq & -\varphi_{0, \delta} \int_{\Omega}\left(f^{0}\left(x, \bar{y}_{\delta}(x), u(x)\right)-f^{0}\left(x, \bar{y}_{\delta}(x), \bar{u}_{\delta}(x)\right)\right. \\
& \left.+f_{y}^{0}\left(x, \bar{y}_{\delta}(x), \bar{u}_{\delta}(x)\right) Y_{\delta}(x)\right) d x+\left\langle\varphi_{\delta}(\cdot), F^{\prime}\left(\bar{y}_{\delta}(\cdot)\right) Y_{\delta}(\cdot)\right\rangle_{\mathcal{Y}^{*}, \mathcal{Y}} .
\end{aligned}
$$

Copyright (c) by SIAM. Unauthorized reproduction of this article is prohibited. 
On the other hand, by (4.18) and the definition of the subdifferential, we have $\psi_{0, \delta} \leq 0$,

$$
\left|\psi_{0, \delta}\right|^{2}+\left\|\varphi_{\delta}(\cdot)\right\|_{\mathcal{Y}^{*}}^{2}=1,
$$

and

$$
\left\langle\varphi_{\delta}(\cdot), \eta-F\left(\bar{y}_{\delta}(\cdot)\right)\right\rangle_{\mathcal{Y}^{*}, \mathcal{Y}} \leq 0 \quad \forall \eta \in E .
$$

Finally, letting $\delta \rightarrow 0^{+}$, along a subsequence, we have

$$
\left(\psi_{0, \delta}, \varphi_{\delta}(\cdot)\right) \rightarrow\left(\bar{\psi}_{0}, \bar{\varphi}(\cdot)\right), \quad \text { weakly* in } \mathbb{R} \times \mathcal{Y}^{*} .
$$

Then $\bar{\psi}_{0} \leq 0$, and it follows from (4.19) that

$$
\begin{aligned}
0 \leq-\bar{\varphi}_{0} \int_{\Omega}( & f^{0}(x, \bar{y}(x), u(x))-f^{0}(x, \bar{y}(x), \bar{u}(x)) \\
& \left.+f_{y}^{0}(x, \bar{y}(x), \bar{u}(x)) Y(x)\right) d x+\left\langle\bar{\varphi}(\cdot), F^{\prime}(\bar{y}(\cdot)) Y(\cdot)\right\rangle_{\mathcal{Y}^{*}, \mathcal{Y}}
\end{aligned}
$$

where $Y(\cdot)$ is the solution to the linearized equation (3.16). Then by duality we obtain

$$
\begin{aligned}
& H\left(x, \bar{y}(x), \bar{\psi}_{0}, \bar{\psi}(x), \nabla \bar{y}(x), \nabla \bar{\psi}(x), \bar{u}(x)\right)-H\left(x, \bar{y}(x), \bar{\psi}_{0}, \bar{\psi}(x), \nabla \bar{y}(x), \nabla \bar{\psi}(x), v\right) \\
& \geq \Phi\left(A(x, v)-A(x, \bar{u}), A(x, v), \nabla \bar{y}(x), \nabla \bar{\psi}(x), e_{1}\right) \quad \forall v \in U, \quad \text { a.e. } x \in \Omega,
\end{aligned}
$$

with $H$ being defined by (4.13). Moreover, we get (4.10) from (4.23).

On the other hand, (4.8) follows from (4.20) and the finite codimensionality of $F^{\prime}(\bar{y}(\cdot)) \mathcal{R}-E$ in $\mathcal{Y}$, while (4.9) follows from (4.21).

5. Some examples. In this section, we present some examples.

Example 5.1. Let $n \geq 2, U=[1,2]$. Consider the controlled system

$$
\left\{\begin{array}{l}
-\nabla \cdot(u(x) \nabla y(x))=1 \quad \text { in } \Omega, \\
\left.y\right|_{\partial \Omega}=0
\end{array}\right.
$$

and the cost functional

$$
J(u(\cdot))=\frac{1}{2} \int_{\Omega}\left[|y(x)-1|^{2}+u(x)^{2}-6 u(x)\right] d x .
$$

Now, suppose $\bar{u}(\cdot)$ is an optimal control. Let $\bar{y}(\cdot)$ be the corresponding optimal state and let $\bar{\psi}(\cdot)$ be the solution of the adjoint equation

$$
\left\{\begin{array}{l}
-\nabla \cdot(\bar{u}(x) \nabla \bar{\psi}(x))=1-\bar{y}(x) \quad \text { in } \Omega, \\
\left.\bar{\psi}\right|_{\partial \Omega}=0 .
\end{array}\right.
$$

By (1.10), for almost all $x \in \Omega$,

$$
\begin{aligned}
& \frac{v^{2}-6 v}{2}-\frac{\bar{u}(x)^{2}-6 \bar{u}(x)}{2}+(v-\bar{u}(x))\langle\nabla \bar{y}(x), \nabla \bar{\psi}(x)\rangle \\
& \geq \frac{(v-\bar{u}(x))^{2}}{v} \frac{|\nabla \bar{y}(x)||\nabla \bar{\psi}(x)|+\langle\nabla \bar{y}(x), \nabla \bar{\psi}(x)\rangle}{2} \quad \forall v \in U .
\end{aligned}
$$

Copyright $@$ by SIAM. Unauthorized reproduction of this article is prohibited. 
Hence, for almost all $x \in \Omega$ and for all $v \in U=[1,2]$,

$$
\begin{aligned}
0 & \leq \frac{(v-\bar{u}(x))^{2}}{v}(|\nabla \bar{y}(x)||\nabla \bar{\psi}(x)|+\langle\nabla \bar{y}(x), \nabla \bar{\psi}(x)\rangle) \\
& \leq v^{2}-6 v-\bar{u}(x)^{2}+6 \bar{u}(x)+2(v-\bar{u}(x))\langle\nabla \bar{y}(x), \nabla \bar{\psi}(x)\rangle \\
& =v^{2}-2(3-\langle\nabla \bar{y}(x), \nabla \bar{\psi}(x)\rangle) v+2(3-\langle\nabla \bar{y}(x), \nabla \bar{\psi}(x)\rangle) \bar{u}(x)-\bar{u}(x)^{2} \\
& =[v-(3-\langle\nabla \bar{y}(x), \nabla \bar{\psi}(x)\rangle)]^{2}-[\bar{u}(x)-(3-\langle\nabla \bar{y}(x), \nabla \bar{\psi}(x)\rangle)]^{2} .
\end{aligned}
$$

Thus, we must have

$$
\bar{u}(x)= \begin{cases}2 & \text { if }\langle\nabla \bar{y}(x), \nabla \bar{\psi}(x)\rangle \leq 1, \\ 3-\langle\nabla \bar{y}(x), \nabla \bar{\psi}(x)\rangle & \text { if } 1<\langle\nabla \bar{y}(x), \nabla \bar{\psi}(x)\rangle<2, \\ 1 & \text { if }\langle\nabla \bar{y}(x), \nabla \bar{\psi}(x)\rangle \geq 2 .\end{cases}
$$

We claim that $\Omega_{0}=\{x \in \Omega \mid 1<\langle\nabla \bar{y}(x), \nabla \bar{\psi}(x)\rangle<2\}$ has zero measure. In fact, we have

$$
\bar{u}(x)=3-\langle\nabla \bar{y}(x), \nabla \bar{\psi}(x)\rangle \in(1,2), \quad \text { a.e. } x \in \Omega_{0} .
$$

Thus, by (5.5), for almost all $x \in \Omega_{0}$ and for all $v \in U$,

$$
\begin{aligned}
& \frac{(v-\bar{u}(x))^{2}}{v}(3-\bar{u}(x))=\frac{(v-\bar{u}(x))^{2}}{v}\langle\nabla \bar{y}(x), \nabla \bar{\psi}(x)\rangle \\
& \quad \leq[v-(3-\langle\nabla \bar{y}(x), \nabla \bar{\psi}(x)\rangle)]^{2}-[\bar{u}(x)-(3-\langle\nabla \bar{y}(x), \nabla \bar{\psi}(x)\rangle)]^{2} \\
& \quad=(v-\bar{u}(x))^{2} .
\end{aligned}
$$

Consequently,

$$
3-\bar{u}(x) \leq v \quad \forall v \in U=[1,2]
$$

By taking $v=1$ we end up with

$$
\bar{u}(x) \geq 2, \quad \text { a.e. } x \in \Omega_{0} .
$$

Combining the above with (5.6), we see that $\Omega_{0}$ has zero measure and $\bar{u}(\cdot)$ is a bangbang control.

Note that if we use (1.12) instead of $(1.10)$, we are not able to get that $\bar{u}(\cdot)$ is bang-bang.

Now, let us consider another example.

Example 5.2. Let $n \geq 2$, and let $U$ be a separable metric space. $R>0$ and $\Omega=B_{R}(0)$. Let the state equation and cost functional be defined by

$$
\left\{\begin{array}{l}
-\nabla \cdot(a(u(x)) \nabla y(x))=1 \quad \text { in } \Omega, \\
\left.y\right|_{\partial \Omega}=0
\end{array}\right.
$$

and

$$
J(u(\cdot))=\int_{\Omega} f^{0}(y(x), u(x)) d x
$$

Copyright (c) by SIAM. Unauthorized reproduction of this article is prohibited. 
where $a: U \rightarrow[\lambda, \Lambda]$ is a continuous function for some $0<\lambda<\Lambda$. When controls are radial functions, for notation simplicity, we denote $r=|x|$ and write $y(x)=y(r)$, $\psi(x)=\psi(r), u(x)=u(r)$, etc. Then the equation in (5.8) becomes

$$
-\nabla \cdot\left(a(u(r)) y^{\prime}(r) \frac{x}{r}\right)=1 .
$$

If we denote $z(r)=a(u(r)) y^{\prime}(r)$, then

$$
\begin{aligned}
& \nabla \cdot\left(a(u(r)) y^{\prime}(r) \frac{x}{r}\right)=\nabla \cdot\left(z(r) \frac{x}{r}\right)=z^{\prime}(r)\left\langle\frac{x}{r}, \frac{x}{r}\right\rangle+z(r) \sum_{i=1}^{n}\left(\frac{x_{i}}{r}\right)_{x_{i}} \\
& =z^{\prime}(r)+z(r) \sum_{i=1}^{n}\left[\frac{1}{r}-\frac{x_{i}^{2}}{r^{2}}\right]=z^{\prime}(r)+\frac{n-1}{r} z(r) .
\end{aligned}
$$

This implies that (5.10) is equivalent to the following:

$$
z^{\prime}(r)+\frac{n-1}{r} z(r)=-1
$$

Since we should have $y^{\prime}(0)=0$, leading to $z(0)=0$, thus

$$
z(r)=-\frac{r}{n} \text {. }
$$

Hence, (5.8) becomes

$$
\left\{\begin{array}{l}
y^{\prime}(r)=-\frac{r}{n a(u(r))}, \quad r \in[0, R], \\
y(R)=0
\end{array}\right.
$$

and (5.9) becomes

$$
J(u(\cdot))=\int_{0}^{R} r^{n-1} f^{0}(y(r), u(r)) d r .
$$

Now, if a radial function $\bar{u}(x)=\bar{u}(r)$ satisfies

$$
J(\bar{u}(\cdot))=\inf _{u \in \mathcal{U}_{R}} J(u(\cdot)),
$$

where

$$
\mathcal{U}_{R} \equiv\{w: \Omega \rightarrow U \mid w \text { is measurable and radial }\},
$$

then such an optimal control problem is essentially a problem for an ODE. By classical results, we have that for almost all $r \in[0, R]$,

$$
\begin{aligned}
\bar{\varphi}(r) \frac{r}{n a(v)} & +r^{n-1} f^{0}(\bar{y}(r), v) \\
& \geq \bar{\varphi}(r) \frac{r}{n a(\bar{u}(r))}+r^{n-1} f^{0}(\bar{y}(r), \bar{u}(r)) \quad \forall v \in U,
\end{aligned}
$$

with $\bar{\varphi}(\cdot)$ being the solution of

$$
\left\{\begin{array}{l}
\bar{\varphi}^{\prime}(r)=r^{n-1} f_{y}^{0}(\bar{y}(r), \bar{u}(r)), \quad r \in[0, R], \\
\bar{\varphi}(0)=0 .
\end{array}\right.
$$

Copyright $@$ by SIAM. Unauthorized reproduction of this article is prohibited. 
We may rewrite (5.16) as

$$
\begin{aligned}
f^{0}(\bar{y}(r), v)-f^{0}(\bar{y}(r), \bar{u}(r))+\frac{\bar{\varphi}(r)}{n r^{n-2}}\left[\frac{1}{a(v)}-\right. & \left.\frac{1}{a(\bar{u}(r))}\right] \geq 0 \\
& \forall v \in U, 0<r<R .
\end{aligned}
$$

Next, suppose $\bar{u}(x)=\bar{u}(r)$ is also an optimal control of the original problem, i.e.,

$$
J(\bar{u}(\cdot))=\inf _{u \in \mathcal{U}} J(u(\cdot)) .
$$

Then by Theorem 1.1, for almost all $x \in \Omega$,

$$
\begin{aligned}
& f^{0}(\bar{y}(r), v)-f^{0}(\bar{y}(r), \bar{u}(r))+(a(v)-a(\bar{u}(x))) \bar{y}^{\prime}(r) \bar{\psi}^{\prime}(r) \\
& \geq \frac{(a(v)-a(\bar{u}(r)))^{2}}{a(v)} \frac{\left|\bar{y}^{\prime}(r) \bar{\psi}^{\prime}(r)\right|+\bar{y}^{\prime}(r) \bar{\psi}^{\prime}(r)}{2} \\
& =\frac{(a(v)-a(\bar{u}(r)))^{2}}{a(v)}\left[\bar{y}^{\prime}(r) \bar{\psi}^{\prime}(r)\right]^{+} \quad \forall v \in U,
\end{aligned}
$$

where $[a]^{+}=\max \{a, 0\}$ and $\bar{\psi}(r)=\bar{\psi}(x)$ is the solution of the adjoint equation

$$
\left\{\begin{array}{l}
-\nabla \cdot(a(\bar{u}(x)) \nabla \bar{\psi}(x))=-f_{y}^{0}(\bar{y}(x), \bar{u}(x)) \quad \text { in } \Omega, \\
\left.\bar{\psi}\right|_{\partial \Omega}=0 .
\end{array}\right.
$$

To compare (5.16) and (5.19), we first note that since all the functions involved in (5.20) are radial, we see that (5.20) is equivalent to the following:

$$
\left\{\begin{array}{l}
-\nabla \cdot\left(a(\bar{u}(r)) \bar{\psi}^{\prime}(r) \frac{x}{r}\right)=-f_{y}^{0}(\bar{y}(r), \bar{u}(r)), \quad 0 \leq r \leq R \\
\bar{\psi}(R)=0
\end{array}\right.
$$

Now, let

$$
\varphi(r)=r^{n-1} a(\bar{u}(r)) \bar{\psi}^{\prime}(r), \quad r \in[0, R] .
$$

Then

$$
\nabla \cdot\left(a(\bar{u}(r)) \bar{\psi}^{\prime}(r) \frac{x}{r}\right)=\nabla \cdot\left(\varphi(r) \frac{x}{r^{n}}\right)=\frac{\varphi^{\prime}(r)}{r^{n-1}}+\varphi(r) \nabla \cdot \frac{x}{r^{n}}=\frac{\varphi^{\prime}(r)}{r^{n-1}} .
$$

On the other hand, we should have $\bar{\psi}^{\prime}(0)=0$, which yields $\varphi(0)=0$. Hence, (5.21) becomes

$$
\left\{\begin{array}{l}
\varphi^{\prime}(r)=r^{n-1} f_{y}^{0}(\bar{y}(r), \bar{u}(r)), \quad 0 \leq r \leq R \\
\varphi(0)=0 .
\end{array}\right.
$$

Comparing the above with $(5.17)$, we see that $\varphi(\cdot)=\bar{\varphi}(\cdot)$. Consequently, taking into account (5.14),

$$
\begin{aligned}
\frac{\bar{\varphi}(r)}{n r^{n-2}}\left[\frac{1}{a(v)}-\frac{1}{a(\bar{u}(r))}\right] & =\frac{r a(\bar{u}(r)) \bar{\psi}^{\prime}(r)}{n} \frac{a(\bar{u}(r))-a(v)}{a(v) a(\bar{u}(r))} \\
& =-a(\bar{u}(r)) \bar{y}^{\prime}(r) \bar{\psi}^{\prime}(r) \frac{a(\bar{u}(r))-a(v)}{a(v)}
\end{aligned}
$$

Copyright $@$ by SIAM. Unauthorized reproduction of this article is prohibited. 
Hence, (5.16) is equivalent to

$$
\begin{aligned}
& f^{0}(\bar{y}(r), v)-f^{0}(\bar{y}(r), \bar{u}(r))+(a(v)-a(\bar{u}(x))) \bar{y}^{\prime}(r) \bar{\psi}^{\prime}(r) \\
& \geq a(\bar{u}(r)) \bar{y}^{\prime}(r) \psi^{\prime}(r) \frac{a(\bar{u}(r))-a(v)}{a(v)}+(a(v)-a(\bar{u}(r))) \bar{y}^{\prime}(r) \bar{\psi}^{\prime}(r) \\
& =\frac{(a(v)-a(\bar{u}(r)))^{2}}{a(v)} \bar{y}^{\prime}(r) \bar{\psi}^{\prime}(r) \quad \forall v \in U .
\end{aligned}
$$

Clearly, (5.19) implies (5.26). Thus, we see that our theorem is stronger than the classical one in some sense when one considers the above radial problem.

Example 5.3. Consider

$$
\left\{\begin{array}{l}
-\nabla \cdot(u(x) A(x) \nabla y(x))=f(x, y(x)) \quad \text { in } \Omega \\
\left.y\right|_{\partial \Omega}=0
\end{array}\right.
$$

with cost functional

$$
J(u(\cdot))=\int_{\Omega} f^{0}(x, y(x)) d x .
$$

We assume that $\Omega \subseteq \mathbb{R}^{n}$ is a bounded domain with a smooth boundary $\partial \Omega, n \geq 2$, $\Lambda>\lambda>0, u(x) \in U \triangleq[\lambda, \Lambda]$, and $A: \Omega \rightarrow \mathcal{S}_{+}^{n}$ is uniformly positive definite. Then

$$
H(x, y, \psi, \xi, \eta, v)=f(x, y)-f^{0}(x, y)-v\langle A(x) \xi, \eta\rangle .
$$

Suppose $\bar{u}(\cdot)$ is an optimal control. Then the adjoint equation is

$$
\left\{\begin{array}{l}
-\nabla \cdot(\bar{u}(x) A(x) \nabla \bar{\psi}(x))=f_{y}(x, \bar{y}(x)) \bar{\psi}(x)-f_{y}^{0}(x, \bar{y}(x)) \quad \text { in } \Omega \\
\left.\bar{\psi}\right|_{\partial \Omega}=0
\end{array}\right.
$$

and the maximum condition reads

$$
\begin{aligned}
& -[\bar{u}(x)-v]\left\langle A(x)^{\frac{1}{2}} \nabla \bar{y}(x), A(x)^{\frac{1}{2}} \nabla \bar{\psi}(x)\right\rangle \\
& \begin{aligned}
\geq \frac{1}{2 v}[\bar{u}(x)-v]^{2}( & \left|A(x)^{\frac{1}{2}} \nabla \bar{y}(x)\right|\left|A(x)^{\frac{1}{2}} \nabla \bar{\psi}(x)\right| \\
& \left.+\left\langle A(x)^{\frac{1}{2}} \nabla \bar{y}(x), A(x)^{\frac{1}{2}} \nabla \bar{\psi}(x)\right\rangle\right) .
\end{aligned}
\end{aligned}
$$

Let

$$
\begin{aligned}
G \triangleq\left\{x \in \Omega|| A(x)^{\frac{1}{2}} \nabla \bar{y}(x) \mid\right. & \left.\left|A(x)^{\frac{1}{2}} \nabla \bar{\psi}(x)\right|>0\right\} \\
& =\{x \in \Omega \mid \nabla \bar{y}(x) \neq 0, \nabla \bar{\psi}(x) \neq 0\} .
\end{aligned}
$$

It is clear that on the set $\Omega \backslash G$, the maximum condition is trivially true and $\bar{u}(\cdot)$ can take any value in $[\lambda, \Lambda]$. We now would like to look at $\bar{u}(\cdot)$ on the set $G$. To this end, we denote

$$
\left\{\begin{array}{l}
G^{+}=\{x \in G \mid\langle A(x) \nabla \bar{y}(x), \nabla \bar{\psi}(x)\rangle>0\} \\
G^{0}=\{x \in G \mid\langle A(x) \nabla \bar{y}(x), \nabla \bar{\psi}(x)\rangle=0\} \\
G^{-}=\{x \in G \mid\langle A(x) \nabla \bar{y}(x), \nabla \bar{\psi}(x)\rangle<0\} .
\end{array}\right.
$$

Copyright $@$ by SIAM. Unauthorized reproduction of this article is prohibited. 
Then on $G^{0},(5.31)$ becomes

$$
0 \geq \frac{1}{v}[\bar{u}(x)-v]^{2}\left|A(x)^{\frac{1}{2}} \nabla \bar{y}(x)\right|\left|A(x)^{\frac{1}{2}} \nabla \bar{\psi}(x)\right|,
$$

which means $G^{0}$ has zero measure since $\Lambda>\lambda$.

On the other hand, (5.31) implies

$$
-[\bar{u}(x)-v]\langle A(x) \nabla \bar{y}(x), \nabla \bar{\psi}(x)\rangle \geq 0 .
$$

Thus,

$$
\bar{u}(x)= \begin{cases}\Lambda, & x \in G^{-} \\ \lambda, & x \in G^{+}\end{cases}
$$

Choosing

$$
v= \begin{cases}\lambda & \text { if } x \in G^{-} \\ \Lambda & \text { if } x \in G^{+}\end{cases}
$$

in (5.31), we get

$$
\begin{array}{r}
\Lambda\left(\left|A(x)^{\frac{1}{2}} \nabla \bar{y}(x)\right|\left|A(x)^{\frac{1}{2}} \nabla \bar{\psi}(x)\right|-|\langle A(x) \nabla \bar{y}(x), \nabla \bar{\psi}(x)\rangle|\right) \\
\leq \lambda\left(\left|A(x)^{\frac{1}{2}} \nabla \bar{y}(x)\right|\left|A(x)^{\frac{1}{2}} \nabla \bar{\psi}(x)\right|+|\langle A(x) \nabla \bar{y}(x), \nabla \bar{\psi}(x)\rangle|\right), \\
\text { a.e. } x \in G
\end{array}
$$

since $G^{0}$ has zero measure.

It is interesting that $G^{0}$ has zero measure, optimal control $\bar{u}(\cdot)$ has to be bangbang on the set $G^{+} \cup G^{-}$, and (5.34) is the necessary condition for optimal control in addition to (5.33).

Acknowledgment. The authors thank the anonymous referees for their helpful suggestions which led to the current, much improved version of the paper.

\section{REFERENCES}

[1] G. Allaire, Homogenization and two-scale convergence, SIAM J. Math. Anal., 23 (1992), pp. $1482-1518$.

[2] A. Bensoussan, J. L. Lions, and G. Papanicolaou, Asymptotic Analysis for Periodic Structures, North-Holland, Amsterdam, 1978.

[3] J. Casado-Díaz, J. Couce-Calvo, and J. D. Martín-Gómez, Optimality conditions for nonconvex multistate control problems in the coefficients, SIAM J. Control Optim., 43 (2004), pp. 216-239.

[4] J. Casado-Díaz, J. Couce-Calvo, and J. D. Martín-Gómez, Relaxation of a control problem in the coefficients with a functional of quadratic growth in the gradient, SIAM J. Control Optim., 47 (2008), pp. 1428-1459.

[5] E. CASAS, Optimal control in coefficients of elliptic equations with state constraints, Appl. Math. Optim., 26 (1992), pp. 21-37.

[6] I. Ciuperca, M. El Alaoui Talibi, and M. Jai, On the optimal control of coefficients in elliptic problems. Application to the optimization of the head slider, ESAIM Control Optim. Calc. Var., 11 (2005), pp. 102-121.

[7] H. Gao And X. LI, Necessary conditions for optimal control of elliptic systems, J. Austral. Math. Soc. Ser. B, 41 (2000), pp. 542-567.

Copyright $@$ by SIAM. Unauthorized reproduction of this article is prohibited. 
[8] X. Li and J. Yong, Optimal Control Theory for Infinite-Dimensional Systems, Birkhäuser, Boston, 1995.

[9] F. Murat and L. Tartar, Calculus of variations and homogenization, in Collection d'tudes d'electricit de France, rewritten in Topics in the Mathematical Modelling of Composite Materials, Progr. Nonlinear Differential Equations Appl. 31, L. Cherkaev and R. V. Kohn, eds., Birkhäuser, Boston, 1998, pp. 139-174.

[10] U. Raitums AND W. H. Schmidt, On necessary optimal conditions for optimal control problems governed by elliptic systems, Optimization, 54 (2005), pp. 149-160.

[11] S. Y. SerovajSKY, Sequential extension in the problem of control in coefficients for elliptic-type equations, J. Inverse Ill-Posed Probl., 11 (2003), pp. 523-536.

[12] R. K. TAgiyev, Optimal control by the coefficients of a parabolic equation, Trans. Acad. Sci. Azerb. Ser. Phys.-Tech. Math. Sci., 24 (2004), pp. 247-256.

[13] A. Zygmund, Trigonometric Series, 3rd ed., Cambridge University Press, Cambridge, UK, 2002.

Copyright (c) by SIAM. Unauthorized reproduction of this article is prohibited. 